A. KODAMA

KODAI MATH. J.

12 (1989), 257-269

\title{
A CHARACTERIZATION OF CERTAIN DOMAINS WITH GOOD BOUNDARY POINTS IN THE SENSE OF GREENE-KRANTZ
}

\author{
By AKIO KODAMA
}

\section{Introduction.}

For a domain $D$ in $C^{n}$, we denote by $\operatorname{Aut}(D)$ the group of all biholomorphic automorphisms of $D$ onto itself and write $\partial D$ (resp. $\bar{D}$ ) for the boundary (resp. closure) of $D$.

In a recent work [6], Greene and Krantz introduced the notions of good or bad boundary points in connection with Rosay-type theorems for weakly pseudoconvex boundary points. For example, consider the weakly pseudoconvex domain

$$
\Omega_{0}=\left\{\left(z_{1}, z_{2}\right) \in C^{2} ;\left|z_{1}\right|^{4}+\left|z_{2}\right|^{4}<1\right\}
$$

and a bounded weakly pseudoconvex domain $\Omega$ in $C^{2}$ such that

(1) there is a point $z^{\circ}$ of $\partial \Omega \cap \partial \Omega_{o}$;

(2) $\Omega \cap U^{\circ}=\Omega_{o} \cap U^{\circ}$ for some open neighborhood $U^{\circ}$ of $z^{\circ}$ in $C^{2}$.

Then the point $z^{\circ}$ is a typical example of bad boundary points of $\Omega_{0}$ in their sense. And they conjecture the following [6; Sect. 13]:

The point $z^{\circ}$ is also a bad boundary point of $\Omega$, that is, the domain $\Omega$ cannot have any $A u t(\Omega)$-orbits accumulating at $z^{0}$.

Clearly this is based on the well-known fact that $\operatorname{Aut}\left(\Omega_{0}\right)$ is a compact Lie group consisting of the holomorphic transformations $\left(z_{1}, z_{2}\right) \rightarrow\left(e^{\sqrt{-1} s} z_{\sigma(1)}, e^{\sqrt{-1}} z_{\sigma(2)}\right)$ ( $s, t \in \boldsymbol{R}$ and $\sigma$ being permutations of $\{1,2\})$ and hence no Aut $\left(\Omega_{o}\right)$-orbit accumulates at $z^{0} \in \partial \Omega_{0}$. Now, generalizing the domain $\Omega_{0}$, we investigate in this paper the weakly pseudoconvex domain

$$
E\left(p_{1}, \cdots, p_{n}\right)=\left\{\left(z_{1}, \cdots, z_{n}\right) \in \boldsymbol{C}^{n} ;\left|z_{1}\right|^{2 p_{1}}+\cdots+\left|z_{n}\right|^{2 p_{n}}<1\right\},
$$

where $p_{1}, \cdots, p_{n}$ are positive integers.

Our main purpose of this paper is to prove the following theorem, from which it follows in particular that the conjecture $(*)$ above is, in fact, true:

Received March 8, 1988; revised January 10, 1989 
THEOREM. Let $D$ be a bounded domain in $\boldsymbol{C}^{n}(n>1)$ with a point $z^{0}=$ $\left(z_{1}^{o}, \cdots, z_{n}^{o}\right) \in \partial D$. After renumbering the coordinates if necessary, we assume that :

(1) There are integers $k \geqq 0, p_{\alpha}>1(k+1 \leqq \alpha \leqq n)$ and an open neighborhood $U^{o}$ of $z^{0}$ in $\boldsymbol{C}^{n}$ such that

(i) $z^{0} \in \partial E\left(1, \cdots, 1, p_{k+1}, \cdots, p_{n}\right)$, and

(ii) $D \cap U^{o}=E\left(1, \cdots, 1, p_{k+1}, \cdots, p_{n}\right) \cap U^{o}$,

here it is of course understood that $E\left(1, \cdots, 1, p_{k+1}, \cdots, p_{n}\right)=B^{n}$, the unit ball in $\boldsymbol{C}^{n}$, if $k=n$.

(2) $\#\left\{i ; z_{i}^{0} \neq 0,1 \leqq i \leqq n\right\}=j$, where \# denotes the number of elements contained in the set.

(3) The point $z^{\circ}$ is a good boundary point of $D$ in the sense of Greene and Krantz [6], that is, there exist a point $k^{\circ} \in D$ and a sequence $\left\{\varphi_{\nu}\right\}$ in Aut $(D)$ such that $\lim _{\nu \rightarrow \infty} \varphi_{\nu}\left(k^{o}\right)=z^{0}$.

Then we have $1 \leqq j \leqq k$ and $D=E\left(1, \cdots, 1, p_{k+1}, \cdots, p_{n}\right)$ as sets. In particular, if $z_{1}^{o} \cdots z_{n}^{o} \neq 0$, then $D$ is precisely the unit ball $B^{n}$.

As an immediate consequence of this, we obtain the following:

COROLlARY. For arbitrary integers $p_{1}, \cdots, p_{n} \geqq 2$, any bounded domain $D$ in $\boldsymbol{C}^{n}$ with a point $z^{0} \in \partial D \cap \partial E\left(p_{1}, \cdots, p_{n}\right)$ near which $\partial D$ coincides with $\partial E\left(p_{1}, \cdots, p_{n}\right)$ cannot have any Aut $(D)$-orbits accumulating at $z^{0}$.

Here it should be noted that any smoothness or pseudoconvexity of $D$ away from $z^{0}$ are not assumed in our Theorem. Moreover, in the special case when $D$ is a bounded domain in $C^{n}$ with $C^{n+1}$-smooth boundary, $\left(p_{1}, \cdots, p_{n-1}, p_{n}\right)=$ $(1, \cdots, 1, m)$ with $m>1$ and $z^{0}=(1,0, \cdots, 0)$ in the Theorem, Greene and Krantz [5] has shown that $D$ is biholomorphically equivalent to the model space $E(1, \cdots, 1, m)$.

After some preliminaries in section 1, the proof of the Theorem will be given in section 2. Our proof here is based on the normal family technique as in [9] and some extension theorems of holomorphic mappings due to Rudin [13], Forstnerič and Rosay [4]. In the final section 3, we shall discuss whether the model space $E\left(1, \cdots, 1, p_{k+1}, \cdots, p_{n}\right)$ can be replaced by any homogeneous bounded (symmetric) domain in our Theorem.

The author would like to thank Professors Steven G. Krantz, Robert E. Greene and Kang-Tae Kim for their useful comments on the subject of this paper. The author would also like to express his thanks to the referee for valuable comments.

\section{Preliminaries.}

In this section we shall recall the structure of the domain $E\left(p_{1}, \cdots, p_{n}\right)$ and some results on extensions of holomorphic mappings. 
For later use of concrete description of biholomorphic automorphisms of $E\left(p_{1}, \cdots, p_{n}\right)$, we begin with recalling the structure of $\operatorname{Aut}\left(E\left(p_{1}, \cdots, p_{n}\right)\right)$. Denoting by $M(r, s)$ the set of all $r \times s$ complex matrices for positive integers $r$ and $s$, we consider the closed Lie subgroup $S U(m, 1)$ of $G L(m+1 ; \boldsymbol{C})$ $(1 \leqq m \leqq n)$ consisting of all matrices

$$
\gamma=\left(\begin{array}{cc}
A & b \\
c & d
\end{array}\right) ; \begin{array}{ll}
A \in M(m, m), & b \in M(m, 1) \\
c \in M(1, m), & d \in M(1,1)
\end{array}
$$

satisfying the relations

$$
{ }^{t} \bar{A} A-{ }^{t} \bar{c} c=E_{m}, \quad{ }^{t} \bar{b} b-|d|^{2}=-1, \quad{ }^{t} \bar{b} A=\bar{d} c \quad \text { and } \quad \operatorname{det} \gamma=1,
$$

where $E_{m}$ is the unit matrix of degree $m$. Moreover, we set

$$
T^{n-m}=\left\{\left(\xi_{m+1}, \cdots, \xi_{n}\right) \in C^{n-m} ;\left|\xi_{\alpha}\right|=1, m+1 \leqq \alpha \leqq n\right\} .
$$

Then, for each $\gamma \in S U(m, 1)$ represented as in $(1.1)$ and each $\xi=\left(\xi_{m+1}, \cdots, \xi_{n}\right) \in$ $T^{n-m}$, we define the transformation $\psi(\gamma, \xi)$ by

$$
\psi(\gamma, \xi):\left\{\begin{array}{l}
z^{\prime} \longmapsto\left(A z^{\prime}+b\right) /\left(c z^{\prime}+d\right), \\
z_{\alpha} \longmapsto \xi_{\alpha} z_{\alpha} /\left(c z^{\prime}+d\right)^{1 / p_{\alpha}}, \quad m+1 \leqq \alpha \leqq n
\end{array}\right.
$$

for $z^{\prime} \in \boldsymbol{C}^{m}$ and $\left(z_{m+1}, \cdots, z_{n}\right) \in \boldsymbol{C}^{n-m}$ (think $z^{\prime}$ as column vector). Using the equality $\left|c z^{\prime}+d\right|^{2}-\left|A z^{\prime}+b\right|^{2}=1-\left|z^{\prime}\right|^{2}$ for all $z^{\prime} \in C^{m}$, one can check easily that each $\psi(\gamma, \xi)$ gives rise to a biholomorphic automorphism of $E\left(1, \cdots, 1, p_{m+1}, \cdots\right.$, $\left.p_{n}\right)$. Moreover, we have the following:

TheOREM A. The domain $E\left(p_{1}, \cdots, p_{n}\right)$ has the following properties

(1.3) $E\left(p_{1}, \cdots, p_{n}\right)$ is a geometrically convex bounded domain in $\boldsymbol{C}^{n}$. In particular, it is taut in the sense of $W u$ [16]; [2], [8].

(1.4) For an arbitrary point $x=\left(x_{1}, \cdots, x_{n}\right) \in \partial E\left(p_{1}, \cdots, p_{n}\right)$, there exists a local holomorphic peaking function $h_{x}$ for $x$ of $E\left(p_{1}, \cdots, p_{n}\right)$. (Consider, for example, the function $h_{x}(z)=\left[2-\sum_{i=1}^{n}\left(z_{\imath}\right)^{p_{i}} \cdot\left(\bar{x}_{\imath}\right)^{p_{i}}\right]^{-1}$ defined near $x$.)

(1.5) Assume that $1 \leqq p_{1} \leqq \cdots \leqq p_{n}$ and $1 \leqq q_{1} \leqq \cdots \leqq q_{n}$. Then $E\left(p_{1}, \cdots, p_{n}\right)$ is biholomorphically equivalent to $E\left(q_{1}, \cdots, q_{n}\right)$ if and only if $p_{i}=q_{\imath}(1 \leqq i \leqq n)$ [11], [15].

(1.6) Assume that $p_{i} \geqq 2(m+1 \leqq i \leqq n)$. Then $\partial E\left(1, \cdots, 1, p_{m+1}, \cdots, p_{n}\right)(0 \leqq m \leqq n)$ is not strictly pseudoconvex precisely at point $z=\left(z_{1}, \cdots, z_{n}\right)$ with $z_{m+1} \cdots z_{n}$ $=0$.

(1.7) Assume that $m \geqq 1$. Then Aut $\left(E\left(1, \cdots, 1, p_{m+1}, \cdots, p_{n}\right)\right)$ contains the Lie subgroup 


$$
G\left(m ; p_{m+1}, \cdots, p_{n}\right)=\left\{\phi(\gamma, \xi) ; \gamma \in S U(m, 1), \xi \in T^{n-m}\right\}
$$

consisting of all transformations defined in (1.2). In particular, for a given sequence $\left\{x^{\nu}\right\}$ in $E\left(1, \cdots, 1, p_{m+1}, \cdots, p_{n}\right)$ converging to a boundary point

$$
x^{o}=\left(x_{1}^{o}, \cdots, x_{n}^{o}\right) \text { with }\left(x_{m+1}^{o}, \cdots, x_{n}^{o}\right)=(0, \cdots, 0),
$$

there exists a sequence $\left\{\phi_{\nu}\right\}$ in $G\left(m ; p_{m+1}, \cdots, p_{n}\right)$ such that

$$
\psi_{\nu}\left(x^{\nu}\right)=\left(0, \cdots, 0, y_{m+1}^{\nu}, \cdots, y_{n}^{\nu}\right), \quad 0 \leqq y_{m+1}^{\nu}, \cdots, y_{n}^{\nu}<1
$$

for all $\nu[7],[14],[9]$.

In 1974, Alexander [1] has shown that certain kinds of holomorphic mappings defined near boundary points of $B^{n}$ must extend to biholomorphic automorphisms of $B^{n}(n>1)$. This was later extended by Rudin to the following:

THEOREM B (Rudin [13]). Assume that $n>1$, and that

(1) $\Omega_{1}$ and $\Omega_{2}$ are connected open subsets of $B^{n}$;

(2) for $j=1,2, \Gamma$, is an open subset of $\partial B^{n}$ such that $\Gamma_{j} \subset \partial \Omega_{j}$;

(3) $f$ is a biholomorphic mapping from $\Omega_{1}$ onto $\Omega_{2}$, and

(4) there is a point $x^{0} \in \Gamma_{1}$, not a limit point of $B^{n} \cap \partial \Omega_{1}$, and a sequence $\left\{x^{i}\right\}$ in $\Omega_{1}$, converging to $x^{o}$, such that $\left\{f\left(x^{i}\right)\right\}$ converges to a point $y^{o} \in \Gamma_{2}$, not a limit point of $B^{n} \cap \partial \Omega_{2}$.

Then there exists $\Phi \in A u t\left(B^{n}\right)$ such that $\Phi(z)=f(z)$ for all $z \in \Omega_{1}$.

In a recent paper [4], Forstnerič and Rosay obtained an interesting theorem on the continuous boundary extension of proper holomorphic mappings. According to [4], we introduce Condition $(P)$ as follows: Let $D$ be a domain in $\boldsymbol{C}^{n}$. Then a point $x^{0} \in \partial D$ satisfies Condition $(P)$ if $\partial D$ is of class $C^{1+\varepsilon}$ near $x^{0}$ for some $\varepsilon>0$ and if there exist a continuous negative plurisubharmonic function $\rho$ on $D$ and a neighborhood $U$ of $x^{o}$ in $C^{n}$ such that

$$
\rho(z) \geqq-c d(z, \partial D), \quad z \in D \cap U
$$

for some constant $c>0$, where $d(z, \partial D)=\inf \{|z-w| ; w \in \partial D\}$.

As noted by themselves, for a domain $D$ in $\boldsymbol{C}^{n}$ we have the following:

(1.8) All $C^{2}$-smooth strictly pseudoconvex boundary points of $D$ satisfy Condition $(\mathrm{P})$;

(1.9) if $\rho$ is a $C^{2}$-smooth plurisubharmonic function on a domain $\Omega \subset C^{n}$, $D=\{z \in \Omega ; \rho(z)<0\}$ and $\bar{D}$ is compact in $\Omega$, then each point $x^{0} \in \partial D$ at which $d \rho\left(x^{0}\right) \neq 0$ satisfies Condition $(\mathrm{P})$.

Now we can state their main result as follows: 
THEOREM C (Forstnerič and Rosay [4]). Let $f: D_{1} \rightarrow D_{2}$ be a proper holomorphic mapping of a domain $D_{1} \subset C^{n}$ onto a bounded domain $D_{2} \subset C^{n}(n \geqq 1)$ and assume that Condition $(P)$ is satisfied for a point $x^{0} \in \partial D_{1}$. If there exists a sequence $\left\{x^{i}\right\} \subset D_{1}$ such that $\lim _{i \rightarrow \infty} x^{i}=x^{0}$, the limit $\lim _{i \rightarrow \infty} f\left(x^{i}\right)=y^{0} \in \partial D_{2}$ exists, and $\partial D_{2}$ is $C^{2}$-smooth strictly pseudoconvex at $y^{\circ}$, then $f$ extends to a Hölder continuous mapping with the exponent $1 / 2$ on a neighborhood of $x^{0}$ in $\bar{D}_{1}$.

\section{Proof of the Theorem.}

Throughout this section, we use the following notation: For given integers $m_{1}, \cdots, m_{n} \geqq 1, \Pi_{\left(m_{1}, \cdots, m_{n}\right)}: \boldsymbol{C}^{n} \rightarrow \boldsymbol{C}^{n}$ will denote the proper holomorphic mapping defined by

$$
\Pi_{\left(m_{1}, \cdots, m_{n}\right)}\left(z_{1}, \cdots, z_{n}\right)=\left(\left(z_{1}\right)^{m_{1}}, \cdots,\left(z_{n}\right)^{m_{n}}\right)
$$

for $\left(z_{1}, \cdots, z_{n}\right) \in C^{n}$. Let $f: M \rightarrow N$ be a mapping from a set $M$ into a set $N$ and $S$ a subset of $M$. Then the restriction of $f$ to $S$ will be denoted by $f \mid S: S \rightarrow N$.

2.1. A Lemma. By using the same technique as in the proof of $[9$; Theorem I], we shall first prove the following:

LEMMA. The domain $D$ is biholomorphically equivalent either to $B^{n}$ or to $E\left(1, \cdots, 1, p_{l+1}, \cdots, p_{n}\right)$, where $1 \leqq \max \{k, j\} \leqq l \leqq n-1$.

Proof. We set $E=E\left(1, \cdots, 1, p_{k+1}, \cdots, p_{n}\right)$ for the sake of simplicity. Without loss of generality, we may assume that $\left\{\varphi_{\nu}\right\}$ converges uniformly on compact subsets of $D$ to the constant mapping $C_{z^{0}}: D \rightarrow C^{n}$ defined by $C_{z o}(z)=z^{0}$ for all $z \in D$. Indeed, this can be seen easily by using the fact that $D$ is a bounded domain in $\boldsymbol{C}^{n}$ and there exists a local holomorphic peaking function for $z^{0}=\lim _{\nu \rightarrow \infty} \varphi_{\nu}\left(k^{0}\right) \in \partial D \cap \partial E$. Fix a family of relatively compact subdomains $D_{\mu}$ of $D$ such that

$$
D=\bigcup_{\mu=1}^{\infty} D_{\mu} \supset \cdots \supset D_{\mu+1} \supset D_{\mu} \supset \cdots \supset D_{1} \ni k^{o}
$$

and choose an integer $\mu \geqq 1$ arbitrarily. Then, since $\varphi_{\nu}(z) \rightarrow z^{0}$ uniformly on $D_{\mu}$, there exists an integer $\nu(\mu)$ such that

$$
\varphi_{\nu}\left(D_{\mu}\right) \subset D \cap U^{o}=E \cap U^{\circ} \quad \text { for all } \nu \geqq \nu(\mu),
$$

where $U^{o}$ is the neighborhood of $z^{o}$ appearing in the Theorem.

Assume first $k=n$, so that $E=B^{n}$. Then $z^{0}$ is a $C^{2}$-smooth strictly pseudoconvex boundary point of $D$; and consequently, by a result of Rosay [12] $D$ is biholomorphically equivalent to $B^{n}$. Thus, it is sufficient to prove the Lemma when $0 \leqq k \leqq n-1$. We have now two cases to consider. 
Case I. $\left(z_{k+1}^{o}, \cdots, z_{n}^{o}\right)=(0, \cdots, 0)$.

First of all, being a point of $\partial E, z^{0}=\left(z_{1}^{o}, \cdots, z_{n}^{o}\right) \neq(0, \cdots, 0)$ and so $1 \leqq j \leqq k$ in this case. By virtue of (1.7), there exists a sequence $\left\{\phi_{\nu}\right\}$ in $\operatorname{Aut}(E)$ such that each point $\phi_{\nu}\left(\varphi_{\nu}\left(k^{0}\right)\right)$ can be expressed as

$$
\phi_{\nu}\left(\varphi_{\nu}\left(k^{0}\right)\right)=\left(0, \cdots, 0, u_{k+1}^{\nu}, \cdots, u_{n}^{\nu}\right) .
$$

Now define biholomorphic mappings $f^{\nu}: D_{\mu} \rightarrow E$ from $D_{\mu}$ into $E$ by

$$
f^{\nu}(z)=\phi_{\nu}\left(\varphi_{\nu}(z)\right), \quad z \in D_{\mu} \quad \text { for all } \quad \nu \geqq \nu(\mu) .
$$

Then, after taking a subsequence and changing the notation if necessary, we obtain the following two cases:

(A) $f^{\nu}\left(k^{0}\right) \rightarrow u^{0}$ for some point $u^{0} \in E$, and

(B) $f^{\nu}\left(k^{o}\right) \rightarrow u^{o}=\left(0, \cdots, 0, u_{k+1}^{o}, \cdots, u_{n}^{o}\right)$ for some point $u^{o} \in \partial E$.

In case (A) we would like to show that $D$ is biholomorphically equivalent to $E$. To do this, recall first $E$ is a taut domain by (1.3). Then, the normality of $\left\{f^{\nu}\right\}$, combined with the fact $f^{\nu}\left(k^{0}\right) \rightarrow u^{o} \in E$, guarantees that some subsequence of $\left\{f^{\nu}\right\}$ converges uniformly on compact subsets to a holomorphic mapping $f(\mu): D_{\mu} \rightarrow E$. Since $\mu$ was arbitrary and $\left\{D_{\mu}\right\}$ increases to $D$ monotonously, by the usual diagonal argument we can assume that $\left\{f^{\nu}\right\}$ itself converges uniformly on $D_{\mu}$ to the holomorphic mapping $f(\mu)$ for all $\mu=1,2, \cdots$. Accordingly we can define a holomorphic mapping $f: D \rightarrow E$ by $f(z)=f(\mu)(z)$, $z \in D_{\mu}$ for $\mu=1,2, \cdots$.

Setting $E_{\nu}=\phi_{\nu}\left(E \cap U^{0}\right)=\phi_{\nu}\left(D \cap U^{o}\right)$ for all $\nu$, we consider the holomorphic mappings $g^{\nu}: E_{\nu} \rightarrow D$ defined by

$$
g^{\nu}(z)=\varphi_{\nu}^{-1}\left(\phi_{\nu}^{-1}(z)\right), \quad z \in E_{\nu} \quad \text { for all } \nu .
$$

Then it is clear that

$$
g^{\nu} \circ f^{\nu}=\operatorname{id}_{D_{\mu}} \text { and } f^{\nu} \circ\left(g^{\nu} \mid f^{\nu}\left(D_{\mu}\right)\right)=\operatorname{id}_{f^{\nu}\left(D_{\mu}\right)}
$$

for all $\nu \geqq \nu(\mu), \mu=1,2, \cdots$. Let $E^{\prime}$ be an arbitrary subdomain of $E$ with compact closure. Then, by means of the concrete description of $\psi_{\nu}$ as in (1.2), one can see that $\phi_{\nu}^{-1}\left(E^{\prime}\right) \subset E \cap U^{o}=D \cap U^{\circ}$ for all sufficiently large $\nu$. Passing to a subsequence if necessary, we can therefore assume that $\left\{g^{\nu}\right\}$ converges uniformly on every compact subset of $E$ to a holomorphic mapping $g: E \rightarrow \bar{D} \subset C^{n}$. Once $g(E) \subset D$ is shown, the equations (2.3) imply that $g \circ f=\mathrm{id}_{D}$ and $f \circ g=\mathrm{id}_{E}$; consequently, $f$ gives a biholomorphic mapping from $D$ onto $E$. Thus we have only to show that $g(E) \subset D$. To this end, take a subdomain $E^{\prime}$ of $E$ with compact closure in $E$ such that $f\left(\bar{D}_{1}\right) \cup f^{\nu}\left(\bar{D}_{1}\right) \subset E^{\prime}$ for all $\nu \geqq \nu_{0}$, where $D_{1}$ is the domain appearing in (2.1) and $\nu_{0}$ is a large integer. Then, for any point $z \in D_{1}$, there are a sequence $\left\{x^{i}\right\} \subset E^{\prime}$ and a subsequence $\left\{g^{\nu}\right\} \subset\left\{g^{\nu}\right\}$ such that $g^{\nu}\left(x^{i}\right)=z$ for all $i$ and $x^{i} \rightarrow x^{o}$ for some point $x^{0} \in \bar{E}^{\prime}$. Hence $z=\lim _{i \rightarrow \infty} g^{\nu} i\left(x^{i}\right)=$ $g\left(x^{o}\right) \in g(E)$, and accordingly $D_{1} \subset g(E)$. On the other hand, being the local 
uniform limit of regular holomorphic mappings $g^{\nu}$, the mapping $g$ is either regular on $E$ or the Jacobian determinant of $g$ vanishes identically on $E$. But, $g(E)$ contains the non-empty open set $D_{1}$. Hence we conclude that $g: E \rightarrow C^{n}$ is regular on $E$ and so $g(E) \subset D$ by [3; Lemma 0] or [10; p. 79], completing the proof in case $(\mathrm{A})$.

In case $(\mathrm{B}): u^{o}=\left(0, \cdots, 0, u_{k+1}^{o}, \cdots, u_{n}^{o}\right) \in \partial E$, we wish to show that $D$ is biholomorphically equivalent either to $B^{n}$ or to $E\left(1, \cdots, 1, p_{l+1}, \cdots, p_{n}\right)$, where $1 \leqq \max \{k, j\}<l \leqq n-1$. Notice first that

$$
\left(u_{k+1}^{o}, \cdots, u_{n}^{o}\right) \neq(0, \cdots, 0)
$$

in case (B). Thus the proof will be divided into two cases as follows:

$$
\text { (B-1) } u_{k+1}^{o} \cdots u_{n}^{o} \neq 0 \text { and (B-2) } u_{k+1}^{o} \cdots u_{n}^{o}=0 \text {. }
$$

In case (B-1) we claim that $D$ is biholomorphically equivalent to $B^{n}$. To prove our claim, choose an open neighborhood $W$ of $u^{o}$ in $\boldsymbol{C}^{n}$ so small that the restriction

$$
\Pi:=\prod_{\left(1, \ldots, 1, p_{k+1}, \ldots, p_{n}\right)} \mid W: W \rightarrow \Pi(W)
$$

is a biholomorphic mapping. This can be always achieved, since all $u_{k+1}^{o}, \cdots, u_{n}^{o} \neq 0$. Then $\Pi(W \cap E)=\Pi(W) \cap B^{n}$ and

$$
\Pi\left(f^{\nu}\left(k^{0}\right)\right) \longrightarrow \Pi\left(u^{0}\right) \in \partial B^{n} \text { as } \nu \rightarrow \infty .
$$

In view of homogeneity of $B^{n}$, there exists a sequence $\left\{\Psi_{\nu}\right\}$ in Aut $\left(B^{n}\right)$ such that

$$
\Psi_{\nu}\left(\Pi\left(f^{\nu}\left(k^{o}\right)\right)\right)=0 \in B^{n} \text { for all sufficiently large } \nu,
$$

where $o$ stands for the origin of $\boldsymbol{C}^{n}$. On the other hand, the existence of a local holomorphic peaking function for $u^{\circ} \in \partial E$ guarantees again that

$$
f^{\nu}\left(D_{\mu}\right) \subset W \cap E \quad \text { for all } \nu \geqq \tilde{\nu}(\mu),
$$

where $\tilde{\nu}(\mu)$ are large integers depending on $\mu=1,2, \cdots$. Accordingly one can define holomorphic mappings $F^{\nu}: D_{\mu} \rightarrow B^{n}$ by setting

$$
F^{\nu}(z)=\Psi_{\nu}\left(\Pi\left(f^{\nu}(z)\right)\right), \quad z \in D_{\mu} \quad \text { for all } \nu \geqq \tilde{\nu}(\mu) .
$$

Since $B^{n}$ is taut and $F^{\nu}\left(k^{o}\right)=o \in B^{n}$ for all $\nu \geqq \tilde{\nu}(\mu), \mu=1,2, \cdots$, we may assume by taking a subsequence if necessary that $\left\{F^{\nu}\right\}$ converges uniformly on compact subsets to a holomorphic mapping $F: D \rightarrow B^{n}$. Here we would like to show that $F$ is a biholomorphic mapping from $D$ onto $B^{n}$. To this end, let us choose a relatively compact subdomain $B^{\prime}$ of $B^{n}$ arbitrarily. Then, taking (1.2) and (2.4) into account, one can see that

$$
\Psi_{\nu}^{-1}\left(B^{\prime}\right) \subset \Pi(W) \cap B^{n} \text { and } \psi_{\nu}^{-1}(W \cap E) \subset E \cap U^{o}=D \cap U^{o}
$$


for all sufficiently large $\nu$, and hence obtain a sequence of holomorphic mappings $G^{\nu}: B^{\prime} \rightarrow D$ defined by

$$
G^{\nu}=\varphi_{\nu}^{-1} \circ \psi_{\nu}^{-1} \circ \Pi^{-1} \circ\left(\Psi_{\nu}^{-1} \mid B^{\prime}\right) \quad \text { for all large } \nu .
$$

Since $B^{\prime}$ is arbitrary, we may assume that $\left\{G^{\nu}\right\}$ converges uniformly on compact subsets to a holomorphic mapping $G: B^{n} \rightarrow \bar{D} \subset C^{n}$. It remains to prove that $G\left(B^{n}\right) \subset D, F \circ G=\mathrm{id}_{B^{n}}$ and $G \circ F=\mathrm{id}_{D^{\circ}}$. But this can be done with exactly the same argument as in the proof of case (A).

In case $(\mathrm{B}-2): u_{k+1}^{o} \cdots u_{n}^{o}=0$, we may rename the indices so that for some $m, k+1 \leqq m \leqq n-1$, one has

$$
u_{k+1}^{o} \cdots u_{m}^{o} \neq 0, \text { while } u_{m+1}^{o}=\cdots=u_{n}^{o}=0 .
$$

Accordingly, the restriction

$$
\Pi:=\Pi_{\left(1, \ldots, 1, p_{k+1}, \ldots, p_{m}, 1, \ldots, 1\right)} \mid W: W \rightarrow \Pi(W)
$$

is biholomorphic on some open neighborhood $W$ of $u^{0}$ in $\boldsymbol{C}^{n}$ and hence

$$
\begin{aligned}
& \Pi(W \cap E)=\Pi(W) \cap E\left(1, \cdots, 1, p_{m+1}, \cdots, p_{n}\right) \\
& \Pi\left(f^{\nu}\left(k^{o}\right)\right) \rightarrow \Pi\left(u^{o}\right)=:\left(v_{1}^{o}, \cdots, v_{n}^{o}\right) \in \partial E\left(1, \cdots, 1, p_{m+1}, \cdots, p_{n}\right)
\end{aligned}
$$

with $\left(v_{m+1}^{o}, \cdots, v_{n}^{o}\right)=(0, \cdots, 0)$. Taking (1.7) into account and passing to a subsequence if necessary, we may assume that

$$
w^{\nu}:=\Psi_{\nu}\left(\Pi\left(f^{\nu}\left(k^{0}\right)\right)\right)=\left(0, \cdots, 0, w_{m+1}^{\nu}, \cdots, w_{n}^{\nu}\right), \quad \nu=1,2, \cdots
$$

for some sequence $\left\{\Psi_{\nu}\right\}$ in $\operatorname{Aut}\left(E\left(1, \cdots, 1, p_{m+1}, \cdots, p_{n}\right)\right)$. If $\left\{w^{\nu}\right\}$ accumulates at some point $w^{0} \in E\left(1, \cdots, 1, p_{m+1}, \cdots, p_{n}\right)$, we conclude by the same reasoning as in case (A) that $D$ is biholomorphically equivalent to $E\left(1, \cdots, 1, p_{m+1}, \cdots, p_{n}\right)$. If a subsequence $\left\{w^{\nu}\right\} \subset\left\{w^{\nu}\right\}$ can be chosen so that $w^{\nu i \rightarrow w^{o}}=\left(0, \cdots, 0, w_{m+1}^{o}, \cdots, w_{n}^{o}\right)$ $\in \partial E\left(1, \cdots, 1, p_{m+1}, \cdots, p_{n}\right)$ for some point $w^{\circ}$, the same situation as in case (B) occurs, but with $m>k$. Thus, repeating this process, we obtain eventually that $D$ is holomorphically equivalent either to $B^{n}$ or to $E\left(1, \cdots, 1, p_{l+1}, \cdots, p_{n}\right)$, where $1 \leqq \max \{k, j\}<m+1 \leqq l \leqq n-1$, as desired.

Case II. $\left(z_{k+1}^{o}, \cdots, z_{n}^{o}\right) \neq(0, \cdots, 0)$.

Assume first $z_{k+1}^{o} \cdots z_{n}^{o} \neq 0$. Then, by a simple modification of the proof in case (B-1), one can see that $D$ is biholomorphically equivalent to $B^{n}$. Moreover, if $z_{k+1}^{o} \cdots z_{n}^{o}=0$, it can be shown in exactly the same way as in case (B-2) that $D$ is biholomorphically equivalent either to $B^{n}$ or to $E\left(1, \cdots, 1, p_{l+1}, \cdots, p_{n}\right)$ with $1 \leqq \max \{k, j\}<l \leqq n-1$, completing the proof of the Lemma.

2.2. Proof of the Theorem. According to the Lemma, we shall divide the proof into two cases as follows: 
Case I. $D$ is biholomorphically equivalent to $B^{n}$.

Since $D$ is homogeneous in this case, we may assume that $z_{1}^{o} \cdots z_{n}^{o} \neq 0$. In particular, $z^{0}$ is a $C^{2}$-smooth strictly pseudoconvex boundary point of $D$ by (1.6). Now, fixing a biholomorphic mapping $F: D \rightarrow B^{n}$ once and for all, we choose a sequence $\left\{z^{i}\right\}$ in $D$ in such a way that

$$
z^{i} \rightarrow z^{o} \text { and } F\left(z^{i}\right) \rightarrow w^{o} \text { for some point } w^{o} \in \partial B^{n} .
$$

Since $\partial B^{n}$ is of course strictly pseudoconvex, both the points $z^{0}$ and $w^{0}$ satisfy Condition $(\mathrm{P})$ by (1.8). Thus, applying Theorem $\mathrm{C}$ to the biholomorphic mapping $F: D \rightarrow B^{n}$ (resp. $F^{-1}: B^{n} \rightarrow D$ ), we obtain a continuous extension

$$
\tilde{F}: V \cap \bar{D} \rightarrow \bar{B}^{n} \quad\left(\text { resp. } H: W \cap \bar{B}^{n} \rightarrow \bar{D}\right)
$$

of $F$ (resp. $F^{-1}$ ), where $V$ (resp. $W$ ) is a sufficiently small open Euclidean ball with center at $z^{o}$ (resp. $w^{o}$ ) in $\boldsymbol{C}^{n}$. Without loss of generality, we may assume that $\tilde{F}(V \cap \bar{D}) \subset W \cap \bar{B}^{n}$ and $V \subset U^{o}$, the neighborhood of $z^{o}$ appearing in the Theorem. Then, by a routine calculation, one can check the following:

(2.7) $\tilde{F}(V \cap \bar{D})$ is a connected open neighborhood of $w^{\circ}$ in $\bar{B}^{n}$;

(2.8) $\tilde{F}: V \cap \bar{D} \rightarrow \tilde{F}(V \cap \bar{D})$ is a homeomorphic mapping.

On the other hand, since all $z_{1}^{o}, \cdots, z_{n}^{o} \neq 0$ by our choice, the holomorphic mapping

$$
\Pi:=\Pi_{\left(1, \ldots, 1, p_{k+1}, \ldots, p_{n}\right)} \mid \tilde{V}: \tilde{V} \rightarrow \Pi(\tilde{V})
$$

is injective for a sufficiently small open neighborhood $\tilde{V}$ of $z^{o}$ in $C^{n}$. After shrinking $V$ if necessary, we can assume that $V \subset \tilde{V}$. Set $G=(\Pi \mid V \cap \bar{D})^{-1}$ and consider the composition

$$
\Psi:=\tilde{F} \circ G: \Pi(V \cap \bar{D}) \rightarrow \tilde{F}(V \cap \bar{D}) .
$$

(Note that $\Pi(V \cap \bar{D}) \cup \tilde{F}(V \cap \bar{D}) \subset \bar{B}^{n}$.) Then, by construction, we have the following :

(2.9) $\Pi(V \cap \bar{D})$ (resp. $\tilde{F}(V \cap \bar{D}))$ is a connected open neighborhood of $\Pi\left(z^{0}\right) \in$ $\partial B^{n}\left(\right.$ resp. $\left.w^{0}=\Psi\left(\Pi\left(z^{o}\right)\right) \in \partial B^{n}\right)$ in $\bar{B}^{n}$;

(2.10) $\Psi \mid \Pi(V \cap D): \Pi(V \cap D) \rightarrow F(V \cap D) \quad($ resp. $\Psi: \Pi(V \cap \bar{D}) \rightarrow \widetilde{F}(V \cap \bar{D})) \quad$ is $\quad$ a biholomorphic (resp. homeomorphic) mapping;

(2.11) $\Psi\left(\Pi(V \cap \bar{D}) \cap \partial B^{n}\right)=\tilde{F}(V \cap \bar{D}) \cap \partial B^{n}$.

Obviously, the hypotheses of Theorem B now hold with

$$
\begin{aligned}
& \Omega_{1}=\Pi(V \cap D), \quad \Omega_{2}=F(V \cap D), \quad x^{o}=\Pi\left(z^{o}\right), \quad y^{o}=w^{o}, \\
& \Gamma_{1}=\Pi(V \cap \partial D), \quad \Gamma_{2}=\tilde{F}(V \cap \partial D) \text { and } f=\Psi,
\end{aligned}
$$

and hence there exists an element $\Phi$ of $\operatorname{Aut}\left(B^{n}\right)$ such that $\Psi=\Phi$ on the non- 
empty open subset $\Pi(V \cap D)$ of $B^{n}$. This combined with the principle of analytic continuation yields that

$$
\Phi^{-1} \circ F\left(z_{1}, \cdots, z_{n}\right)=\left(z_{1}, \cdots, z_{k},\left(z_{k+1}\right)^{p_{k+1}}, \cdots,\left(z_{n}\right)^{p_{n}}\right)
$$

for all $\left(z_{1}, \cdots, z_{n}\right) \in D$, from which we have $D \subset E\left(1, \cdots, 1, p_{k+1}, \cdots, p_{n}\right)$. Note that $\Phi^{-1} \circ F: D \rightarrow B^{n}$ is a biholomorphic mapping from $D$ onto $B^{n}$. Thus, if $\left\{z^{i}\right\}$ is a sequence in $D$ that has no limit point in $D$, then $\left\{\Phi^{-1} \circ F\left(z^{i}\right)\right\}$ has no limit point in $B^{n}$. It follows in particular from this that there exists no boundary point of $D$ in $E\left(1, \cdots, 1, p_{k+1}, \cdots, p_{n}\right)$ and consequently $D=E\left(1, \cdots, 1, p_{k+1}, \cdots\right.$, $\left.p_{n}\right)$. Since $E\left(1, \cdots, 1, p_{k+1}, \cdots, p_{n}\right)$ is now biholomorphically equivalent to $B^{n}=E(1, \cdots, 1)$, we conclude by $(1.5)$ that $k=n$ and $D=E(1, \cdots, 1)=B^{n}$, completing the proof in Case I.

Case II. $\quad D$ is biholomorphically equivalent to $E:=E\left(1, \cdots, 1, p_{l+1}, \cdots, p_{n}\right)$ with $1 \leqq \max \{k, j\} \leqq l \leqq n-1$.

Fixing a biholomorphic mapping $F: D \rightarrow E$ from $D$ onto $E$, we take a point

$$
z^{\prime}=\left(z_{1}^{\prime}, \cdots, z_{n}^{\prime}\right) \in U^{\circ} \cap \partial D \text { with } z_{1}^{\prime} \cdots z_{n}^{\prime} \neq 0 .
$$

There exists a sequence $\left\{z^{i}\right\}$ in $D$ such that

$$
z^{2} \rightarrow z^{\prime} \text { and } w^{2}:=F\left(z^{i}\right) \rightarrow w^{\prime} \in \partial E
$$

for some boundary point $w^{\prime}$. Since $z^{\prime}$ is a $C^{2}$-smooth strictly pseudoconvex boundary point of $D$ by (1.6) and $w^{\prime}$ satisfies Condition (P) by (1.9), the inverse mapping $F^{-1}: E \rightarrow D$ has a continuous extension $H: W \cap \bar{E} \rightarrow \bar{D}$ by Theorem $\mathrm{C}$, where $W$ is an open neighborhood of $w^{\prime}$ in $\boldsymbol{C}^{n}$. Now, keeping the fact $H\left(w^{\prime}\right)=\lim _{\imath \rightarrow \infty} H\left(w^{i}\right)=z^{\prime}$ in mind, we choose an open neighborhood $U^{\prime}$ (resp. $W^{\prime}$ ) of $z^{\prime}$ (resp. $w^{\prime}$ ) in $\boldsymbol{C}^{n}$ in such a way that

(2.12) $U^{\prime} \subset U^{o} \cap\left\{\left(z_{1}, \cdots, z_{n}\right) \in \boldsymbol{C}^{n} ; z_{1} \cdots z_{n} \neq 0\right\}$;

(2.13) $W^{\prime} \subset W$ and $H\left(W^{\prime} \cap \bar{E}\right) \subset U^{\prime}$.

Take a point

$$
w^{\prime \prime}=\left(w_{1}^{\prime \prime}, \cdots, w_{n}^{\prime \prime}\right) \in W^{\prime} \cap \partial E \quad \text { with } \quad w_{1}^{\prime \prime} \cdots w_{n}^{\prime \prime} \neq 0
$$

and set $z^{\prime \prime}=H\left(w^{\prime \prime}\right) \in U^{\prime} \cap \partial D$. Then, both the points $z^{\prime \prime}$ and $w^{\prime \prime}$ are $C^{2}$-smooth strictly pseudoconvex boundary points; and hence they satisfy Condition $(\mathrm{P})$ by (1.8). Therefore, repeating the same argument as in Case I, we obtain an open neighborhood $U^{\prime \prime}$ of $z^{\prime \prime}$ in $C^{n}$ satisfying the following:

(2.14) $U^{\prime \prime} \subset U^{\circ}$ and $U^{\prime \prime} \cap D$ is a connected open subset of $D$;

(2.15) $F$ has a continuous extension $\tilde{F}: U^{\prime \prime} \cap \bar{D} \rightarrow \bar{E}$;

(2.16) $\tilde{F}\left(U^{\prime \prime} \cap \bar{D}\right)$ is an open neighborhood of $w^{\prime \prime}$ in $\bar{E}$;

(2.17) $\tilde{F}: U^{\prime \prime} \cap \bar{D} \rightarrow \tilde{F}\left(U^{\prime \prime} \cap \bar{D}\right)$ is a homeomorphic mapping. 
On the other hand, since $z_{1}^{\prime \prime} \cdots z_{n}^{\prime \prime} \neq 0$ (resp. $w_{1}^{\prime \prime} \cdots w_{n}^{\prime \prime} \neq 0$ ) by our choice, the holomorphic mapping

$$
\Pi:=\Pi_{\left(1, \ldots, 1, p_{k+1}, \ldots, p_{n}\right)}\left(\text { resp. } \tilde{\Pi}:=\Pi_{\left(1, \ldots, 1, p_{l+1}, \ldots, p_{n}\right)}\right)
$$

is injective on some open neighborhood of $z^{\prime \prime}$ (resp. $\left.w^{\prime \prime}\right)$. After shrinking $U^{\prime \prime}$ if necessary, we can therefore assume that:

(2.18) $\Pi \mid U^{\prime \prime}: U^{\prime \prime} \rightarrow \Pi\left(U^{\prime \prime}\right)$ is a biholomorphic mapping;

(2.19) $\tilde{\Pi}$ is biholomorphic on some open neighborhood of $\tilde{F}\left(U^{\prime \prime} \cap \bar{D}\right)$.

Now we set $G=\left(\Pi \mid U^{\prime \prime} \cap \bar{D}\right)^{-1}$ and consider the composition

$$
\Psi:=\tilde{\Pi} \circ \tilde{F} \circ G: \Pi\left(U^{\prime \prime} \cap \bar{D}\right) \longrightarrow \tilde{\Pi} \circ \tilde{F}\left(U^{\prime \prime} \cap \bar{D}\right) .
$$

Then, in exactly the same way as in the proof of Case I, it can be shown that there exists an element $\Phi \in \operatorname{Aut}\left(B^{n}\right)$ such that

$$
\Phi^{-1} \circ \tilde{\Pi} \circ F\left(z_{1}, \cdots, z_{n}\right)=\left(z_{1}, \cdots, z_{k},\left(z_{k+1}\right)^{p_{k+1}}, \cdots,\left(z_{n}\right)^{p_{n}}\right)
$$

for all $\left(z_{1}, \cdots, z_{n}\right) \in D$. This combined with the fact that $\Phi^{-1} \circ \tilde{\Pi} \circ F: D \rightarrow B^{n}$ is a proper holomorphic mapping yields at once that $D=E\left(1, \cdots, 1, p_{k+1}, \cdots, p_{n}\right)$. Finally, since $E\left(1, \cdots, 1, p_{k+1}, \cdots, p_{n}\right)$ is now biholomorphically equivalent to $E\left(1, \cdots, 1, p_{l+1}, \cdots, p_{n}\right)$ and $1 \leqq k \leqq l \leqq n-1$, we conclude by $(1.5)$ that $k=l$; and consequently, $1 \leqq \max \{k, j\} \leqq l=k \leqq n-1$. Therefore we have completed the proof of the Theorem.

q. e. d.

\section{Concluding remarks.}

Remark 1. Let us consider once more the model spaces $E\left(1, \cdots, 1, p_{m+1}, \cdots\right.$, $\left.p_{n}\right)(1 \leqq m \leqq n)$ in this paper or the domains $E(k, \alpha)$ in the previous paper [9]. Then we can see that such model spaces $E$ admit many biholomorphic automorphisms, which fact plays an important role in the proofs of our theorems. So it would be naturally expected that the same conclusion as in the theorems is also true if the model spaces $E$ are bounded homogeneous (symmetric) domains. More precisely speaking, we might hope that the following is possible: Let $E$ be a bounded homogeneous (symmetric) domain in $\boldsymbol{C}^{n}$ and $D$ a bounded domain in $\boldsymbol{C}^{n}$ with a common boundary point $z^{0} \in \partial D \cap \partial E$. Assume that:

(1) $D \cap U^{o}=E \cap U^{o}$ for some open neighborhood $U^{o}$ of $z^{o}$ in $\boldsymbol{C}^{n}$;

(2) there exist a point $k^{\circ} \in D$ and a sequence $\left\{\varphi_{\nu}\right\}$ in $\operatorname{Aut}(D)$ with $\lim _{\nu \rightarrow \infty} \varphi_{\nu}\left(k^{o}\right)=z^{o}$.

Then $D$ is biholomorphically equivalent to $E$ (or more strongly $D=E$ as sets).

Unfortunately, this hope has been dashed by the following:

EXAmple. Denoting by $\Delta$ the unit disc in $\boldsymbol{C}$, we consider the domains $E=\Delta \times \Delta \times \Delta, \quad D=\Delta \times B^{2}$ in $C^{3}$ and the point $z^{0}=(1,0,0) \in \partial E \cap \partial D$. Then we have 
(1) $D$ and $E$ are bounded homogeneous symmetric domains;

(2) $D$ is not biholomorphically equivalent to $E$; and

(3) $D \cap U^{o}=E \cap U^{o}$ for some open neighborhood $U^{o}$ of $z^{o}$ in $C^{3}$.

A crucial difference between the symmetric domain $E$ above and the model spaces considered previously is the fact that $E$ admits no local holomorphic peaking function for the point $z^{0}$.

Remark 2. In the special case of complex two dimension, by modifying the proof of the theorem, one can show that the analogue of our theorem is also valid for more general domain

$$
E\left(p_{1}, p_{2}\right)=\left\{\left(z_{1}, z_{2}\right) \in C^{2} ;\left|z_{1}\right|^{2 p_{1}}+\left|z_{2}\right|^{2 p_{2}}<1\right\}
$$

with arbitrary real numbers $p_{1}, p_{2}>0$.

\section{REFERENCES}

[1] H. Alexander, Holomorphic mappings from the ball and polydiscs, Math. Ann. 209 (1974), 249-256.

[2] T. J. BARTh, Convex domains and Kobayashi hyperbolicity, Proc. Amer. Math. Soc. 79 (1980), 556-558.

[3] J.E. Fornaess and E. L. Stout, Polydiscs in complex manifolds, Math. Ann. 227 (1977), 145-153.

[4] F. Forstnerič AND J.P. Rosay, Localization of the Kobayashi metric and the boundary continuity of proper holomorphic mappings, Math. Ann. 279 (1987), 239-252.

[5] R.E. Greene AND S.G. KRANTZ, Characterizations of certain weakly pseudoconvex domains with non-compact automorphism groups, Lecture Notes in Math. 1268, Springer-Verlag, Berlin, Heidelberg, New York, London, Paris and Tokyo, 1987, 121-157.

[6] R.E. Greene and S.G. KrantZ, Biholomorphic self-maps of domains, Lecture Notes in Math. 1276, Springer-Verlag, Berlin, Heidelberg, New York, London, Paris and Tokyo, 1987, 136-207.

[7] M. IsE, On Thullen domains and Hirzebruch manifolds, I, J. Math. Soc. Japan 26 (1974), 508-522.

[8] P. KIERNAN, On the relations between taut, tight and hyperbolic manifolds, Bull. Amer. Math. Soc. 76 (1970), 49-51.

[9] A. KodAma, Characterizations of certain weakly pseudoconvex domains $E(k, \alpha)$ in $\boldsymbol{C}^{n}$, Tôhoku Math. J. 40 (1988), 343-365.

[10] R. NARAsimhan, Several complex variables, Univ. Chicago Press, Chicago and London, 1971.

[11] I. NARUKI, The holomorphic equivalence problem for a class of Reinhardt domains, Publ. Res. Inst. Math. Sci., Kyoto Univ., 4 (1968), 527-543.

[12] J.P. Rosay, Sur une caractérisation de la boule parmi les domaines de $\boldsymbol{C}^{n}$ par son groupe d'automorphismes, Ann. Inst. Fourier (Grenoble) 29, 4 (1979), 9197.

[13] W. Rudin, Holomorphic maps that extend to automorphisms of a ball, Proc. 
Amer. Math. Soc. 81 (1981), 429-432.

[14] T. SunAdA, Holomorphic equivalence problem for bounded Reinhardt domains, Math. Ann. 235 (1978), 111-128.

[15] S. M. Webster, Biholomorphic mappings and the Bergman kernel off the diagonal, Invent. Math. 51 (1979), 155-169.

[16] H. Wu, Normal families of holomorphic mappings, Acta Math. 119 (1967), 193233.

Department of Mathematics

FACULTy OF SCIENCE

KanAZAWA University

KANAZAWA, 920 JAPAN 